\title{
El procesamiento temporal en la percepción del habla de los niños con dislexia
}

\author{
${ }^{1}$ Universidad de La Laguna \\ ${ }^{2}$ Universidad de Antioquia
}

Rosario Ortiz ${ }^{*}$, Adelina Estévez ${ }^{1}$ y Mercedes Muñetón ${ }^{2}$

\begin{abstract}
Resumen: Numerosos estudios muestran que un déficit fonológico causa la dislexia evolutiva, pero el origen de este déficit continúa siendo un tema controvertido. Este estudio examina si el déficit perceptivo fonológico de los niños con dislexia puede ser explicado por un déficit de procesamiento temporal mediante un diseño que controla las demandas de la tarea y la complejidad lingüística y temporal de los contrastes entre sonidos del habla. Trece niños con dislexia y déficit fonológico (9-11 años de edad) y 13 niños sin dificultades en lectura emparejados en edad fueron evaluados en tareas de juicio de orden temporal (JOT) y en tareas de discriminación igualdiferente (I-D). Las tareas se basan en contrastes de las sílabas /ba/ -/da/ y /fa/-/la/. Analizamos los efectos de la complejidad de los estímulos (similar vs. no similar) y de la tarea (tarea de juicio de orden temporal vs. discriminación). Los niños con dislexia muestran peor rendimiento que los niños sin dificultades en lectura en ambos pares de sílabas. El rendimiento de los niños con dislexia en las tareas JOT fue significativamente inferior que el de los niños del grupo control pero no encontramos diferencias entre los grupos en las tareas I-D. Los hallazgos son discutidos en términos de problemas de procesamiento temporal en la percepción del habla de los niños con dislexia.
\end{abstract}

Palabras clave: dislexia; percepción del habla; procesamiento temporal; procesamiento auditivo rápido, déficit fonológico.

\section{Introducción}

La dislexia se define como una dificultad específica de aprendizaje de origen neurobiológico, que se presenta en niños que han recibido una enseñanza adecuada y tienen una inteligencia normal. Se caracteriza por dificultades en la precisión y la fluidez en el reconocimiento de palabras y por problemas en la descodificación y el deletreo de palabras. Estas dificultades son causadas por un déficit en el componente fonológico del lenguaje (Lyon, Shaywitz, \& Shaywitz, 2003). Se han postulado distintas causas, cognitivas y biológicas, de este trastorno de lectura. No obstante, la teoría dominante es que un déficit en el procesamiento fonológico subyace a la dislexia evolutiva (Ramus, 2004; Ramus et al., 2003). Estas deficiencias en el procesamiento fonológico que presentan las personas con dislexia obstaculizan la comprensión y aplicación de las reglas de correspondencia grafemafonema necesarias para aprender a leer en las lenguas alfabéticas. El déficit fonológico en las personas con dislexia ha sido demostrado por distintos equipos de investigación en diferentes lenguas y utilizando una amplia variedad de tareas. Esta teoría también es apoyada por estudios neuronatómicos y neurofuncionales que revelan que las personas con dislexia muestran una disfunción cerebral en las regiones perisilvianas del hemisferio izquierdo durante la ejecución de tareas que requieren procesamiento fonológico (para una revisión

* Dirección para correspondencia [Correspondence address]: Rosario Ortiz. Facultad de Psicología. Universidad de La Laguna. Campus de Guajara. 38071 La Laguna (Tenerife), España.

E-mail: mrortiz@ull.es
Title: Temporal processing in speech perception of children with dyslexia. Abstract: Numerous studies show that a phonological deficit causes dyslexia, but the origin of this deficit remains a controversial issue. The present study examines whether perceptual phonological deficit in children with dyslexia can be explained by a temporal processing deficit with a design that controls the demands of the task and linguistic complexity and temporal of contrasts between speech sounds. Thirteen children with dyslexia and phonological deficits (9-11 years) and 13 age-matched normal readers were assessed on temporal order judgment tasks (TOJ) and samedifferent discrimination tasks (S-D). The tasks are based on contrasts of the syllables / ba / - / da / and / fa/-/ la /. We analyze the effects of the complexity of the stimuli (similar versus no similar) and the task (temporal order judgment tasks versus discrimination tasks). The children with dyslexia showed lower performance than the control group in both pairs of syllables. The performance of children with dyslexia in the TOJ tasks was significantly lower than the control group but no differences between groups in the S-D tasks were found. The findings are discussed in terms of temporal processing problems in speech perception in children with dyslexia.

Key words: Dyslexia; speech perception; temporal processing; rapid auditory processing; phonological deficit.

ver Shaywitz, Morris, \& Shaywitz, 2008). Esta disfunción consiste en la ausencia o la reducción de la activación en esta región del hemisferio izquierdo, en comparación con las personas sin dificultades en lectura. Además, se observa un incremento de la activación en esta misma región en el hemisferio derecho y en las áreas del córtex prefrontal en los sujetos con dislexia (Backes et al., 2002; Eden, \& Zeffiro, 1998; Temple, 2002).

El interés actual de los investigadores se centra en identificar el origen de este déficit fonológico que presentan las personas con dislexia. Numerosos estudios han apuntado hacia las dificultades en el procesamiento perceptivo de los sonidos del habla. Vandermosten et al. (2011), revisaron 32 estudios de percepción categórica en niños con dislexia. Concluyeron que en el $64 \%$ de los experimentos los individuos con dislexia muestran dificultades en la percepción de las consonantes oclusivas. En varios de estos estudios se usaron como estímulos pares de sílabas que se diferencian en el punto de articulación (p.e., /ba/-/da/), observándose un rendimiento inferior en la percepción de este contraste fonético en el grupo de niños con dislexia frente a los del grupo control (De Gelder, \& Vroomen, 1998; Godfrey, Syrdal-Lasky, Millay, \& Knox, 1981; McArthur, Ellis, Atkinson, \& Coltheart, 2008; Paul, Bott, Heim,Wienbruch, \& Elbert, 2006; Reed, 1989; Steffens, Eilers, Gross-Glenn, \& Jallad, 1992; Werker, \& Tees, 1987). También, los resultados de Maassen, Groenen, Crul, Assman-Hulsmans, and Gabreels (2001) revelaron que los niños con dislexia presentan déficit en las habilidades de discriminación del punto de articulación y de la sonoridad (p.e., /pa/-/ba/). Además, existen evidencias experimentales de que el déficit de percepción 
del habla del grupo de niños con dislexia se manifiesta en cualquier tipo de contraste fonético. Ortiz et al. (2007) encontraron en los niños con dislexia un rendimiento inferior al de los niños sin dificultades en lectura emparejados en edad lectora, en tareas de discriminación de la sonoridad, tareas de discriminación del punto de articulación y tareas de discriminación del modo de articulación (p.e., /ba/-/ma/).

Los niños con dislexia presentan un retraso evolutivo del desarrollo de la percepción del habla durante la educación primaria. Las habilidades de discriminación de las claves fonéticas necesarias para percibir el habla (la sonoridad, el punto y el modo de articulación) se siguen desarrollando durante la educación primaria en los niños sin dificultades lectoras, pero no en los niños con dislexia quienes sólo avanzan en la discriminación del punto de articulación (Ortiz et al., 2008). Además, Serniclaes, Sprenger-Charolles, Carré, and Demonet (2001) mostraron que los participantes con dislexia tienen dificultades en la discriminación de categorías fonéticas, pero tienen mejor rendimiento que los participantes sin dificultades en lectura en las tareas de discriminación de sílabas dentro de la misma categoría. Esto es, los niños con dislexia perciben mejor que los niños del grupo control las diferencias entre las variantes acústicas del mismo fonema (p.e., dos /b/ diferentes). Estos resultados sugieren que la percepción de los individuos con dislexia está basada en alófonos más que en fonemas. Otros estudios han mostrado que los niños con dislexia presentan dificultades para procesar algunas propiedades temporales de los sonidos del habla, por ejemplo, el ritmo. Goswami et al. (2011) evaluaron en distintas lenguas (inglés, español y chino) la habilidad de los niños con dislexia para percibir el ritmo de los sonidos. Los resultados mostraron que los niños con dislexia fueron menos sensibles a los ritmos de las secuencias de sonidos que los niños sin dislexia. También, encontraron que el procesamiento de la clave acústica fundamental para detectar el compás rítmico del habla predice la conciencia fonológica y la lectura. En general, los estudios revisados muestran los niños con dislexia presentan dificultades en el procesamiento perceptivo auditivo.

La mayoría de los estudios que examinan las dificultades en el procesamiento perceptivo auditivo en individuos con dislexia se plantean en torno a dos hipótesis: la hipótesis del déficit de procesamiento temporal y la hipótesis del déficit específico en percepción del habla. La primera hipótesis postula que la dislexia se explica por un déficit general de procesamiento temporal, que se manifiesta en distintos ámbitos, entre ellos, el procesamiento fonológico (Tallal, 1980; Tallal, \& Piercy, 1973, 1974, 1975). Tallal asume que los déficits fonológicos en la dislexia son secundarios a déficits de procesamiento temporal auditivo de bajo nivel. Los déficits de procesamiento temporal alteran la percepción de los elementos acústicos cuando éstos son de corta duración o se caracterizan por transiciones rápidas. Por este motivo, los niños con dislexia tendrían dificultades para procesar los sonidos breves que cambian rápidamente y están ordenados secuencialmente, como las transiciones de los formantes o el ruido espectral asociado a las consonantes oclusivas. En conse- cuencia, los niños con dislexia tendrían problemas para desarrollar las habilidades fonológicas necesarias para establecer las correspondencias entre grafemas y fonemas. Los resultados de varios estudios han apoyado esta hipótesis del déficit de procesamiento temporal (v.g., Farmer, \& Klein, 1995; Habib et al., 2002; Hämäläinen, Salminen, \& Läpanen, 2012; Hari, \& Kiesila, 1996; Kinsbourne, Rufo, Gamzu, Palmer, \& Berliner, 1991; McBride-Chang, 1996; Reed, 1989; Rey, De Martino, Espesser, \& Habib, 2002; Stein, \& McAnally, 1995; Tallal, 1980; Vandermosten, et al., 2011). Sin embargo, otras investigaciones no han encontrado evidencias de un déficit de procesamiento temporal en los participantes con dislexia (v.g., Best, \& Avery, 1999; Bishop, Carylon, Deeks, \& Bishop, 1999; Bradlow et al., 1999; Breier, Gray, Fletcher, Foorman, \& Klaas, 2002; McAnally, Hansen, Cornelissen, \& Stein, 1997; Mody, Studdert-Kennedy, \& Brady,1997; Serniclaes et al., 2001; Share, Jorm, Maclean, \& Matthews, 2002). La segunda hipótesis, la del déficit específico del habla, plantea que las dificultades lectoras se producen por un fallo del sistema lingüístico para discriminar las representaciones fonológicas de los fonemas, más que por un fallo en el procesamiento temporal de señales acústicas (Mody et al., 1997). Esta hipótesis sostiene que la dificultad para derivar representaciones fonológicas de la corriente acústica del habla subyace a los déficits fonológicos de los individuos con dislexia. Mody et al. (1997) hicieron una revisión crítica de los estudios tempranos de Tallal y observaron que en estos estudios se usaban dos sílabas similares fonéticamente para evaluar el procesamiento temporal. El estudio de Mody et al. (1997) demostró que cuando se presentan las tareas de orden temporal con sílabas fonéticamente diferentes los niños con dislexia tienen un rendimiento similar al del grupo control. Ellos concluyeron que los niños de los estudios de Tallal eran incapaces de detectar el orden temporal de las sílabas, no por un déficit de procesamiento temporal, sino por la dificultad para identificar sílabas fonéticamente similares. Esta hipótesis del déficit específico del habla se apoya en los estudios que muestran que los individuos con dislexia no tienen problemas de discriminación auditiva general sino problemas específicos en la percepción de palabras y fonemas (Adlard, \& Hazan, 1998; Blomert, \& Mitterer, 2004; Breier et al., 2002; De Weirdt, 1988; Lieberman, Meskill, Chatillon, \& Schupack, 1985; Mody et al., 1997; Reed, 1989; Schulte-Koerne, Deimel, Bartling, \& Remschmidt, 1999; Tobey, \& Cullen, 1984; Werker, \& Tees, 1987). También avalan esta hipótesis los estudios que mostraron que la percepción del habla hace una contribución significativa a la lectura y a la conciencia fonológica (v.g., Chiappe, Chiappe, \& Siegel, 2001; Csépe, Gyurkócza, \& Osman-Sági, 1998; De Weirdt, 1988; Godfrey et al.,1981; Metsala, 1997; Ortiz y Guzmán, 2003; Reed, 1989; Werker, \& Tees, 1987).

El debate sobre si el origen del déficit fonológico es un déficit específico del habla o un déficit general de procesamiento temporal ha persistido a lo largo de los años, en parte, porque hay datos experimentales que apoyan y rechazan cada una de estas hipótesis. También, otras cuestiones con- 
tribuyen a que el origen del déficit fonológico continúe sin aclararse. La confusión terminológica y conceptual entre procesamiento temporal y procesamiento rápido de estímulos presentados secuencialmente ha estado presente en la investigación en este campo. Mody et al. (1997) plantearon la necesidad de diferenciar entre ambos conceptos. Sólo cuando se presentan dificultades para percibir propiedades temporales de los estímulos (duración, secuencia y ritmo) podemos hablar de déficit de procesamiento temporal. Si las dificultades se presentan en la identificación y discriminación de estímulos cuya duración es muy corta o en la percepción de estímulos con intervalos muy breves entre ellos, entonces se trata de un déficit de procesamiento rápido del habla. La falta de distinción entre estos conceptos ha llevado a interpretar los bajos resultados en tareas de procesamiento rápido como dificultades de procesamiento temporal y ha generado una gran controversia (ver Mody et al., 1997; Rosen, 2003). En algunos estudios (p.e., Cohen-Mimram, 2006) se utiliza como indicador de procesamiento temporal una tarea igualdiferente (I-D) en la que se solicita a niños con y sin dislexia discriminar auditivamente entre pares de sílabas que pueden ser diferenciadas sobre la base de una clave temporal breve (i.e., $/ \mathrm{ba} /-/ \mathrm{pa} /$ ). Este tipo de tarea permite detectar las dificultades de los individuos con dislexia en el procesamiento perceptivo de estímulos rápidos pero no evalúa el procesamiento temporal, ya que requiere un juicio de identidad rápida y no de temporalidad.

Una forma de asegurarse de que se está evaluando el procesamiento temporal es administrando tareas que requieren apreciar las propiedades temporales de los estímulos. Este es el caso de las tareas de juicio de orden temporal (JOT). En estas tareas se presentan dos estímulos auditivos, secuencialmente y a distintos intervalos. Se le pide al oyente identificar el orden en que se han presentado los dos estímulos. En sus estudios iniciales Tallal y colaboradores usaron esta tarea JOT para evaluar el procesamiento temporal y la tarea I-D como una tarea control, porque esta última también tiene un componente perceptivo y otro secuencial, pero no requiere procesamiento temporal (Tallal y Piercy, 1973, 1974, 1975). Los estímulos fueron bien sílabas sintéticas que difieren sólo en una característica fonética $(/ \mathrm{ba} /-/ \mathrm{da} /)$, bien tonos complejos similares en el contenido acústico al contraste de sílabas. Otros estudios también compararon la habilidad de los participantes con dislexia para discriminar sonidos del habla con su habilidad para discriminar sonidos no lingüísticos, análogos al habla. Usaron procedimientos sofisticados para incorporar ciertas características acústicas a los sonidos no lingüísticos. La idea es que estas características acústicas permitan diferenciar los sonidos no lingüísticos tal como se haría con los sonidos del habla. Este paradigma experimental ha sido criticado por algunos investigadores argumentando que el sustrato perceptual del procesamiento de estímulos no lingüísticos no es el mismo que el del procesamiento del habla (Ellis, 2005). También ha sido criticado por los que sostienen que la conexión entre los déficits en las habilidades de procesamiento de sonidos no lingüísticos y las dificultades con los sonidos del habla no ha sido demostrada (Rosen, 2003). Varios estudios que controlaron el tipo de tarea mostraron que los individuos con dislexia presentan dificultades, principalmente, en el procesamiento de los sonidos del habla. Pero los investigadores no controlaron la complejidad de los estímulos, por lo que sus resultados pueden explicarse bien por la existencia de un déficit específico del habla, bien por la mayor complejidad acústica de los estímulos lingüísticos en comparación con los no lingüísticos (Breier et al., 2002; Mody et al., 1997; Ramus et al., 2003; Rosen, \& Manganari, 2001). En consecuencia, para explorar la naturaleza de los déficits de percepción del habla en la dislexia parece necesario usar sonidos naturales del habla y controlar la complejidad de la tarea y la complejidad acústica de los sonidos. El control de los estímulos debería hacerse tanto a nivel lingüístico, como en la dimensión temporal.

El presente estudio se encuadra en el debate sobre si el origen del déficit fonológico es un déficit específico del habla o si subyace a un déficit general de procesamiento temporal. Específicamente, se plantea la hipótesis de que el déficit fonológico de los niños con dislexia se asocia a un déficit de procesamiento temporal. El estudio trata de superar los citados obstáculos metodológicos que podrían explicar la divergencia de resultados que caracterizan esta línea de trabajo. Con este objetivo se plantea un diseño experimental cuidadoso que controla las demandas de la tarea y la complejidad lingüística y temporal de los contrastes entre los sonidos del habla. En esta investigación, se compara el rendimiento de un grupo de niños con dislexia con el de un grupo de niños sin dificultades en lectura en las tareas JOT, que evalúan el procesamiento temporal y en las tareas I-D, que evalúan el procesamiento perceptivo rápido de secuencias de sonidos del habla. Siguiendo a Tallal y colaboradores, entendemos la tarea I-D como una tarea control porque no requiere procesamiento temporal. El trabajo examina la naturaleza del déficit fonológico de los niños con dislexia mediante la comparación del rendimiento en estas tareas entre los dos grupos. La hipótesis del déficit de procesamiento temporal predice que los niños con dislexia presentarán más dificultades que los del grupo control únicamente en las tareas JOT. Si, por el contrario, el rendimiento de los niños con dislexia fuera inferior al del grupo control en las tareas I-D, el déficit fonológico no podría asociarse a dificultades con el procesamiento temporal. En caso de encontrar alguna diferencia entre los grupos hay que asegurarse de que ésta no sea una consecuencia de una dificultad específicamente lingüística con la complejidad del contraste fonético. Por eso, se controla la complejidad de los contrastes de los sonidos del habla a nivel lingüístico: se compara el rendimiento en las tareas cuando se presenta un par de sílabas fonéticamente similares que sólo se diferencian en el punto de articulación (/ba/-/da/) (i.e., contraste de mayor complejidad), con el rendimiento en las tareas cuando se presenta un par de sílabas fonéticamente diferentes (se diferencian en el punto de articulación, el modo de articulación y la sonoridad $(/ \mathrm{fa} /-/ \mathrm{la} /))$. Si los niños con dislexia presentan más dificultades que los niños sin di- 
ficultades en lectura sólo en los pares de sílabas fonéticamente similares, con independencia del tipo de tarea, las diferencias entre los grupos podrían atribuirse a un problema específicamente lingüístico para procesar los fonemas dinámicos (p.e., las consonantes oclusivas). Mientras que si el rendimiento de los niños con dislexia es inferior al del grupo control en ambos tipos de sílabas, con independencia de la tarea, las diferencias entre los grupos no podrían atribuirse a un déficit específico al habla, sino a dificultades con el procesamiento temporal.

\section{Método}

\section{Participantes}

La muestra de estudio estaba formada por 26 niños de Educación Primaria (14 niños y 12 niñas) con un rango de edad de 9 a 11 años. Del total de niños que participaron en la muestra, 13 eran niños sin dificultades en lectura que cursaban $4^{\circ}$ de Primaria con una media de edad de 117.30 $(D T=3.27)$ y 13 niños con dislexia y déficit fonológico con una media de edad de 119.15 (DT=8.21). La muestra de niños con dislexia (CD) estaba formada por niños que acudían a la Unidad de Atención a las Dificultades de Aprendizaje (U.A.D.A.) de la Universidad de La Laguna y contaban con un diagnóstico de dislexia realizado por profesionales. La muestra de alumnos sin problemas de lectura (SD) se obtuvo de una muestra más amplia de alumnos procedentes de un centro concertado.

La identificación de los niños con dislexia se llevó a cabo por un especialista en dificultades de aprendizaje. Los criterios utilizados para el diagnóstico fueron los siguientes: a) PC $\leq 25$ en aciertos en la lectura de pseudopalabras o PC $\geq$ 75 en el tiempo empleado en la lectura de palabras, o PC $\geq$ 75 en el tiempo empleado en la lectura de pseudopalabras; b) CI $\geq 80$ con el fin de no incluir en la muestra niños con déficit intelectual. Los criterios utilizados para la selección de los niños sin dificultades en lectura fueron: a) $\mathrm{PC} \geq 50$ en aciertos en la lectura de palabras y pseudopalabras; b) PC $\leq$ 50 en tiempo de lectura de palabras y pseudopalabras; c) CI $\geq 80$; d) la identificación por parte del profesor como alumno sin problemas en la lectura. Los grupos estaban igualados en edad $[t(24)=3.917 ; p=.59]$; inteligencia $[t(24)=$ -2.555; $p=.12]$ y memoria de dígitos $[t(24)=0.460 ; p=$ .70]. Sin embargo, los niños CD presentaban un rendimiento inferior al del grupo de niños SD en conciencia fonémica [ $t$ $(24)=7.280 ; p=.01$ ] (Ver Tabla 1). En el estudio no participaron niños con necesidades educativas específicas por discapacidad, por trastorno generalizado del desarrollo, por trastornos graves de conducta $\mathrm{u}$ otros problemas tradicionalmente usados como criterios de exclusión para la dislexia. Todos los alumnos que participaron en esta investigación contaron con el consentimiento de sus padres.
Tabla 1. Media y Desviación Típica en edad, C.I., memoria de dígitos y conciencia fonémica.

\begin{tabular}{|c|c|c|c|c|c|}
\hline & \multicolumn{2}{|c|}{$\begin{array}{l}\text { Niños con } \\
\text { dislexia }\end{array}$} & \multicolumn{2}{|c|}{$\begin{array}{l}\text { Niños sin } \\
\text { dificultades } \\
\text { en lectura }\end{array}$} & \multirow[b]{2}{*}{$p$} \\
\hline & $M$ & $D T$ & $M$ & $D T$ & \\
\hline Edad & 119.15 & 8.21 & 117.30 & 3.27 & .59 \\
\hline C.I. & 106.63 & 12.71 & 114.00 & 10.51 & .12 \\
\hline Memoria dígitos & 12.61 & 1.93 & 14.00 & 1.77 & .70 \\
\hline Conciencia fonémica & 9.90 & 2.86 & 12.88 & 1.21 & .01 \\
\hline
\end{tabular}

\section{Instrumentos}

PROLEC-R. Batería de Evaluación de los procesos lectores Revisada (Cuetos, Rodríguez, Ruano y Arribas, 2007): se administró en primer lugar el subtest de identificación de letras con el objetivo de comprobar si los niños conocen todas las letras y posteriormente, los subtests de lectura de palabras y pseudopalabras. Estos subtests requieren la correcta identificación de 40 palabras y 40 pseudopalabras, registrándose el número de aciertos y el tiempo invertido en la lectura de cada listado. La puntuación total se obtiene asignando un punto a cada respuesta correcta y considerando el tiempo invertido en cada subtest.

Factor "g" de Cattell y Cattell (2001): este test estandarizado permite evaluar la capacidad intelectual general mediante tareas no verbales. Se administró la escala 2 (forma A) para escolares de 8 a 14 años. Esta escala está compuesta por cuatro subtests: series, clasificación, comparación y matrices.

Memoria de dígitos: se aplicó la subprueba Test de Dígitos del WISC-IV (Wechsler, 2005) que mide memoria auditiva a corto plazo y memoria de trabajo. En esta tarea el niño repite una serie de números que el evaluador presenta verbalmente. Se comienza con dos dígitos y se va aumentando un dígito más hasta que se produzcan dos fallos consecutivos. En primer lugar, la repetición de la serie se efectúa en el mismo orden de presentación y posteriormente, en orden inverso.

Conciencia fonémica (Jiménez, 1995): consta de cuatro tareas: a) síntesis o reunificación de fonemas para formar palabras; b) aislar el fonema inicial o final de palabras; c) segmentar palabras en fonemas; d) omisión del fonema inicial o final de palabras. Cada tarea incluye 15 palabras que se presentan oralmente y de forma individual. La puntuación de cada tarea se obtiene asignando un punto a cada respuesta correcta. La puntuación total es la media de las puntuaciones obtenidas en las cuatro tareas.

Prueba informatizada de Percepción del Habla (PH): se trata de una prueba informatizada, diseñada para esta investigación, que evalúa la percepción de dos pares de sílabas con diferente complejidad lingüística. Se usa un par de sílabas fonéticamente similar (/ba/ - /da/) donde las sílabas se diferencian sólo en el punto de articulación y otro par no similar (/fa/ /la/). En este último par, las sílabas se diferencian en la sonoridad, el punto de articulación y el modo de articulación. Se presentan cuatro tareas. Dos de ellas son tareas JOT, en estas tareas los niños escuchan los pares de sílabas a través 
de los auriculares y señalan la sílaba que apareció en primer lugar pulsando una tecla. En la tarea 1 los niños oyen los pares de silabas $(/ \mathrm{fa} / \mathrm{-} / \mathrm{la} /)$ y pulsan la tecla $\mathrm{F}$ o L. En la tarea 2, escuchan el par (/ba/ - /da/) y pulsan la tecla B o D. En cada tarea se incluían tres condiciones con intervalos temporales entre las sílabas de cada par de estímulos (ISIs) cortos $(40,150$ y $250 \mathrm{~ms})$ y tres con ISIs largos $(310,400$ y $500 \mathrm{~ms})$. Cada tarea tiene 2 ejemplos con 8 ensayos cada uno en los que reciben retroalimentación positiva o negativa sobre su ejecución. A continuación, se presentan los ensayos experimentales sin retroalimentación, 24 con ISIs cortos y 24 ensayos con ISIs largos. Las otras dos son tareas I-D. La tarea 3 evalúa la habilidad para discriminar pares de sílabas fonéticamente distintas (/fa/ - /la/ y /la/ - /fa/). La tarea 4 evalúa la habilidad para discriminar sílabas fonéticamente similares (/ba/ - /da/ y /da/ - /ba/). En ambas tareas el niño debe indicar si las sílabas que ha escuchado son iguales o no pulsando las teclas S o N. En cada tarea los ítems se presentaban contrabalanceados formando 4 pares de sílabas (p.e., $/ \mathrm{fa} /$ - /fa/; /la/ - /la/, /fa/ - /la/; /la/ - /fa/) con 10 ensayos cada uno. Previamente, realizaban dos ejemplos con 4 ensayos cada uno en los que recibían retroalimentación. El ISI en estas tareas fue de $250 \mathrm{~ms}$. En todas las tareas se mostraba en la pantalla un asterisco $950 \mathrm{~ms}$ antes de la presentación del par de estímulos y el tiempo máximo de respuesta fue $5 \mathrm{~s}$. Todos los estímulos fueron pronunciados por una experta y grabados en el laboratorio de Fonética Experimental de la Universidad de La Laguna en una grabadora digital. Luego la voz natural se pasó de la grabadora al disco duro del ordenador. La ventaja de este método es que se conservan casi todas las características de la voz humana y el ordenador produce una voz inteligible y natural (Van Daal, \& Van Der Leij, 1992). El ordenador registraba el número de aciertos en cada ensayo. La prueba de $\mathrm{PH}$ tiene un alfa de Cronbach .92.

\section{Procedimiento}

Las pruebas fueron administradas por dos psicólogos especialistas en dificultades de aprendizaje. Los niños diagnosticados como niños con dislexia acudían a la U.A.D.A. de la Universidad de La Laguna donde los evaluadores les administraron las pruebas de manera individual. Las pruebas se aplicaron individualmente a la muestra de niños sin dificultades en lectura en un aula cedida por el centro que reunía las condiciones de tranquilidad necesaria para este fin. Para la administración de las pruebas se utilizaron dos sesiones. En la primera sesión los niños realizaron dos tareas de percepción del habla, el conocimiento de letras y la lectura de palabras y pseudopalabras. En la segunda sesión, se administraron las dos tareas restantes de percepción del habla, la prueba de conciencia fonémica, la memoria de dígitos y el test de CI.

\section{Resultados}

Con los datos obtenidos se llevaron a cabo varios análisis. En primer lugar, comparamos la puntuación total en la prueba de $\mathrm{PH}$ entre los grupos de niños con dislexia y niños sin dificultades en lectura. En segundo lugar, comparamos la ejecución de los dos grupos en cada tarea, esto es, por una parte la tarea I-D y por la otra, la tarea JOT. En tercer lugar, contrastamos las dos tareas sólo en los ISIS de $250 \mathrm{~ms}$, dado que ambas tareas tenían en común la utilización de este intervalo temporal entre estímulos. Finalmente, comparamos el rendimiento global de los dos grupos en cada par de sílabas, es decir, por una parte / ba/ - /da/ y por otra / fa/ /la/. Para comparar los grupos llevamos a cabo análisis Ttest. Calculamos los tamaños del efecto con el programa G*power3 (Faul, Erdfelder, Lang, \& Buchner, 2007), en el cual se da un tamaño del efecto según lo propuesto por Cohen. La Tabla 2 muestra la media y la desviación típica en PH total, sílabas y tareas. En percepción de habla total los resultados muestran que los niños con dislexia obtienen menos aciertos que los niños sin dificultades en lectura $[t$ $(24)=-2.57 ; p=.02, \mathrm{~d}=1.01]$. Al comparar cada tarea entre los grupos, los resultados muestran que en la tarea I-D no existen diferencias significativas $[t(24)=-1.48 ; p=.16$, $\mathrm{d}=0.60]$. Sin embargo, en la tarea JOT sí hay diferencias significativas entre los grupos $[t(24)=-2.64, p=.02, \mathrm{~d}=$ 1.15], siendo los niños con dislexia quienes presentan un rendimiento inferior. Cuando se contrastan las dos tareas sólo en los ISIS de $250 \mathrm{~ms}$ (ver Figura 1), los resultados también muestran que en la tarea I-D no hay diferencias significativas entre los grupos $[t(24)=-1.48, p=.16, \mathrm{~d}=0$. 60]. Sin embargo, en la tarea JOT los niños con dislexia producen significativamente menos aciertos $[t(24)=-2.52, p$ $=.02, \mathrm{~d}=.21]$ que los niños sin dificultades en lectura.

Tabla 2. Media de aciertos, desviaciones típicas, $p$ y tamaño del efecto (d) en percepción del habla total (PH), sílabas y tareas en función de los grupos.

\begin{tabular}{|c|c|c|c|c|c|c|}
\hline & \multicolumn{2}{|c|}{$\begin{array}{c}\text { Niños con } \\
\text { dislexia }\end{array}$} & \multicolumn{2}{|c|}{$\begin{array}{c}\text { Niños sin } \\
\text { dificultades en lectura }\end{array}$} & \multirow[b]{2}{*}{$p$} & \multirow[b]{2}{*}{ d } \\
\hline & $M$ & DT & $M$ & $D T$ & & \\
\hline PH & .89 & .11 & .97 & .02 & .02 & 1.01 \\
\hline$/ \mathrm{ba} /-/ \mathrm{da} /$ & .86 & .16 & .96 & .02 & .04 & 0.87 \\
\hline$/ \mathrm{fa} /$ - /la/ & .92 & .06 & .98 & .02 & .01 & 1.34 \\
\hline JOT & .89 & .11 & .98 & .01 & .02 & 1.15 \\
\hline $\mathrm{I}-\mathrm{D}$ & .88 & .16 & .95 & .04 & .16 & 0.60 \\
\hline
\end{tabular}

Al comparar las sílabas estudiadas (/ba/ - /da/ y /fa/ /la/) se observa que los niños CD producen significativamente menos aciertos que los niños SD en el par de sílabas $/ \mathrm{ba} / \mathrm{-} / \mathrm{da} /[t(24)=-2.18 ; p=.04, \mathrm{~d}=0.87]$. En el par de sílabas $/ \mathrm{fa} /-/ \mathrm{la} /$ se observa el mismo patrón, es decir, los niños $\mathrm{CD}$ producen significativamente menos aciertos que los niños SD $[t(24)=-2.98 ; p=.01, \mathrm{~d}=1.34]$. El análisis intragrupo de los contrastes entre sílabas muestra que no hay diferencias en el grupo CD $[t(12)=-1.94 ; p=.07, \mathrm{~d}=$ $0.10]$, mientras que en el grupo SD las puntuaciones en el 
contraste $/ \mathrm{ba} /-/ \mathrm{da} /$ son inferiores a las obtenidas en el par $/ \mathrm{fa} /-/ \mathrm{la} /[t(12)=-2.36 ; p=.03, \mathrm{~d}=1.3]$.

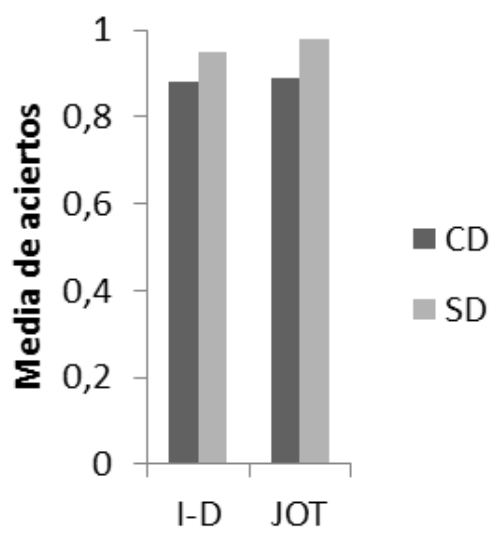

Figura 1. Media de aciertos en las tareas I-D y JOT con el ISI de $250 \mathrm{~ms}$. del grupo de niños con dislexia (CD) y del grupo de niños sin dificultades en lectura (SD).

\section{Discusión}

Este estudio examina si el déficit fonológico de los niños con dislexia puede explicarse por dificultades con el procesamiento temporal. La predicción de la hipótesis del déficit temporal es que los niños con dislexia presentarán más dificultades que los del grupo control únicamente en las tareas JOT. Los resultados se ajustan a esta predicción, ya que el rendimiento de los niños con dislexia es inferior al del grupo control en las tareas JOT, pero ambos grupos no se diferencian en las tareas I-D. Esto ocurre también cuando el ISI entre las sílabas es idéntico en ambas tareas. Es decir, los niños con dislexia que participaron en este estudio, presentan dificultades, únicamente, en la tarea que demanda el procesamiento temporal de los sonidos del habla. Para comprobar que la dificultad en la tarea JOT no es una consecuencia de una dificultad específicamente lingüística con la complejidad del contraste fonético, se analizaron los resultados en función de la complejidad de los contrastes de los sonidos del habla. El rendimiento de los niños con dislexia es inferior al del grupo de niños sin dificultades en lectura en ambos tipos de sílabas, con independencia de la tarea. Esto es, no les afecta la complejidad del contraste fonético. En consecuencia, el bajo rendimiento de los niños con dislexia en la tarea JOT no debe atribuirse a un déficit específico al habla, sino a dificultades con el procesamiento temporal.

Los resultados muestran que los niños con dislexia obtienen puntuaciones inferiores a los niños sin dificultades en lectura en la puntuación total en percepción del habla, lo que revela la presencia de las dificultades fonológicas características de esta población. Los niños con dislexia presentan un rendimiento inferior al de los niños sin dificultades en lectura en las tareas JOT, mientras que no hay diferencias entre los grupos en las tareas I-D. Los resultados en la tarea JOT, en términos generales, son convergentes con otros estudios que también usaron estímulos lingüísticos (De-Martino, Espesser, Rey, \& Habib, 2001; Wolff, Michel, \& Ovrut, 1990). Incluídos los de aquellos estudios que usaron estructuras silábicas y fonemas diferentes, por ejemplo, en el estudio de Rey et al. (2002) encontraron que los niños con dislexia entre 10 y 13 años obtienen un rendimiento menor que los del grupo control en una tarea JOT en la que se contrastaban sílabas con distinta estructura (consonante-consonante-vocal y consonante-vocal). También, son resultados similares a los de Breier et al. (2002) que mostraron que niños de 7.3 a 14.5 años con dislexia presentaban un rendimiento inferior a los niños sin dislexia en la tarea JOT usando los estímulos sintéticos $/ \mathrm{ba} / \mathrm{y} / \mathrm{da} /$. Además, son convergentes con estudios que usaron sonidos no lingüísticos (Laasonen et al., 2012; Pasquini, Corriveau, \& Goswami, 2007).

En esta investigación no encontramos diferencias significativas entre los grupos en la tarea I-D. Lo que indica que el déficit fonológico de los niños con dislexia no se explica por dificultades en la discriminación de las sílabas. Estos resultados son covergentes con los del estudio de Mody et al. (1997) que sugieren que al no existir diferencias significativas entre los grupos de niños con dislexia y niños sin dificultades en lectura en la tarea I-D, los resultados encontrados en el rendimiento de una tarea JOT pueden atribuirse a dificultades en el procesamiento temporal y no a dificultades para discriminar las sílabas. Sin embargo, nuestros resultados no coinciden con otras investigaciones que arrojan diferencias entre los grupos en tareas I-D que emplean sílabas fonéticamente similares en todas las tareas (v.g., CohenMimran,2006; Maassen, et al., 2001; Ortiz et al., 2007). Por ejemplo, Cohen-Mimran (2006) encontró que los niños con dislexia rendían menos que los niños sin dificultades en lectura en tareas I-D con las sílabas naturales /pa/-/ba/. La diferencia con el presente estudio puede radicar en la naturaleza de las sílabas empleadas. Las sílabas usadas en ese estudio son articulatoria y acústicamente diferentes a las usadas aquí. Cohen-Mimran usó sílabas cuyo contraste, desde el punto de vista articulatorio, radica en la vibración de las cuerdas vocales $(/ \mathrm{pa} /-/ \mathrm{ba} /)$, mientras que en el actual estudio se usan sílabas cuyo contraste radica en el punto de articulación (/ba/-/da/). No obstante, Breier et al. (2002) usaron las mismas sílabas que las del presente trabajo en tareas I-D y obtuvieron resultados diferentes. Ellos encontraron que los niños con dislexia presentaban un rendimiento inferior a los niños sin dislexia en la tarea I-D. La diferencia entre los estímulos de ambos trabajos es que aquéllos eran sintéticos y los del presente estudio son naturales. Por tanto, la diferencia en los resultados puede reflejar que el sustrato perceptual del procesamiento de estímulos sintéticos no es el mismo que el del procesamiento de estímulos naturales del habla (Ellis, 2005).

En cuanto a la complejidad lingüística de los contrastes fonéticos, los alumnos con dislexia muestran en este estudio peor rendimiento que los niños sin dificultades en lectura tanto en el procesamiento de contrastes fonéticos complejos $(/ \mathrm{ba} /-/ \mathrm{da} /)$ como en los simples $(/ \mathrm{fa} /-/ \mathrm{la} /)$. Además, en 
los niños con dislexia no se encontraron diferencias significativas al comparar sus puntuaciones entre los dos contrastes, sus puntuaciones no mejoran al disminuir la complejidad del contraste fonético. La dificultad de los niños con dislexia en el par más complejo ( $/ \mathrm{ba} /-/ \mathrm{da} /)$ es un resultado convergente con el de otros estudios que han evaluado la percepción categorial de este par de sílabas en niños con dislexia (Breier et al., 2002; de Gelder, \& Vroomen, 1998 ; Godfrey et al., 1981; Reed, 1989; Rosen, \& Manganari, 2001; Vandermosten et al., 2011; Werker, \& Tees, 1987) y en adultos con dislexia (Dufor, Serniclaes, Sprenger-Charolles, \& Démonet, 2007; Ramus et al., 2003; Steffens et al., 1992; Vandermosten, et al., 2010). Las oclusivas (i.e., /p/, /b/, $/ \mathrm{d} /, / \mathrm{t} /, \ldots)$ son más difíciles de percibir porque ocurren más rápidamente que otras consonantes $(/ \mathrm{f} /, / \mathrm{r} /, / 1 /, .$. (Zhang, \& McBride-Chang, 2010). Esto explica que los niños sin dificultades en lectura tengan peor rendimiento en el $\mathrm{par} / \mathrm{ba} /-/ \mathrm{da} /$ que en el par/fa/-/la/. Pero esto no ocurre en los niños con dislexia, lo que pone de manifiesto que el nivel de complejidad lingüística no afecta a los niños con dislexia. Lo que parece indicar que sus problemas de percepción del habla no son consecuencia de una dificultad específicamente lingüística con la complejidad del contraste fonético.

El análisis de los resultados obtenidos por los niños con dislexia en las dos tareas de percepción del habla muestra que no tienen dificultades para el procesamiento lingüístico de las sílabas sino para procesar propiedades temporales de los sonidos del habla. El análisis de datos en función de la complejidad lingüística de los contrastes fonéticos denota las dificultades de los niños con dislexia en la percepción de los fonemas. Tienen dificultades para percibir contrastes de poca complejidad en las que se presentan fonemas lentos y diferentes en sonoridad, punto y modo de articulación (/fa//la/) y no sólo para percibir contrastes con pares mínimos de consonantes rápidas o dinámicas $(/ \mathrm{ba} /-/ \mathrm{da} /)$. Por tanto, una dificultad lingüística con la complejidad de los contrastes fonéticos no puede ser la causa de las dificultades con la tarea que requiere procesamiento temporal. El tamaño de la muestra de este estudio es pequeño y limita la generalización de estos resultados a otras poblaciones de niños con dislexia. No obstante, hemos de considerar que los resultados obtenidos en este estudio son contrarios a la idea de que un problema en la detección de cambios acústicos rápidos en el habla o una confusión perceptual entre fonemas muy similares produce los déficits en percepción del habla. Como señala Rosen (2003) es posible que las dificultades para procesar propiedades temporales de los sonidos del habla (duración, secuencia, ritmo) obstaculizacen la creación de representaciones fonológicas precisas y estables de los fonemas. En este sentido, los resultados apoyarían la tesis de Zhang and McBride-Chang (2010) en cuanto a que las hipótesis de procesamiento temporal y del déficit específico del habla no son mutuamente excluyentes. Estos autores proponen un modelo en el que el procesamiento temporal impacta sobre la percepción de fonemas y ésta última incide en los tres compo- nentes del procesamiento fonológico implicados en la lectura (conciencia fonológica, memoria verbal y velocidad de nombrado).

El diseño del presente estudio no permite abordar relaciones causales, pero los resultados son convergentes con la hipótesis del déficit de procesamiento temporal. Varios autores han defendido que un déficit básico de procesamiento temporal produce una cascada de efectos, comenzando por problemas en la percepción del habla que causan alteraciones en desarrollo del sistema fonológico, lo que a su vez genera dificultades de aprendizaje en lectura y escritura (Talcott, \& Witton, 2002; Tallal, 1980; Wright et al., 1997). Estas dificultades en el procesamiento temporal suponen diferencias en la percepción del habla que podrían tener un fuerte impacto durante toda la fase del aprendizaje y consolidación del proceso lector. La dificultad en identificar la temporalidad de los sonidos del habla puede dar lugar a categorías fonológicas poco precisas que dificulten la segmentación de las palabras. En consecuencia, parece conveniente que en los programas fonológicos de tratamiento de la dislexia se incluya un entrenamiento en percepción del habla, focalizado en la dimensión temporal.

En este estudio no podemos aclarar si las dificultades en procesamiento temporal encontradas en los niños con dislexia están restringidas únicamente a los sonidos del habla o podría tratarse de un déficit general en el procesamiento temporal de cualquier sonido, porque sólo hemos incluido estímulos lingüísticos. Goswami et al. (2011) mostraron que los niños con dislexia presentan déficits en el procesamiento temporal de sonidos no lingüísticos y que estos déficits están relacionados con sus dificultades fonológicas y con su rendimiento lector. Se necesita un estudio específico que aborde la existencia de dificultades de procesamiento temporal con la misma tarea en estímulos lingüísticos y no lingüísticos y su relación con los déficit fonológicos. Otra línea de trabajo interesante es examinar si los individuos con dislexia también presentan dificultades en el procesamiento temporal de estímulos visuales. Los resultados del estudio meta-analítico de Sánchez-Meca (1985) muestran que el procesamiento de la información visual en los lectores retrasados es más lento que en las personas sin dificultades en lectura cuando la presentación de los estímulos es serial. Por tanto, sería conveniente examinar la percepción de estímulos visuales y auditivos en personas con dislexia para averiguar si las dificultades encontradas en el procesamiento temporal de los sonidos del habla son una manifestación de un déficit de procesamiento temporal más general que afecta a todo tipo de estímulos.

\section{Conclusiones}

Esta investigación aporta algunos datos que sugieren que el déficit fonológico de los niños con dislexia se asocia a dificultades en el procesamiento temporal de los sonidos del habla. Los niños con dislexia mostraron más dificultades que los del grupo control en la tarea que requiere procesamiento temporal, con independencia de la complejidad lingüística 
del contraste fonético. Sin embargo, no se encontraron diferencias entre los grupos en la tarea de discriminación perceptiva. Las dificultades de procesamiento temporal encontradas en los niños con dislexia no se podrían atribuir a la complejidad acústica de los contrastes fonéticos porque estos niños mostraron problemas tanto para procesar fonemas que contienen información secuencial que cambia rápidamente en un breve periodo de tiempo (consonantes oclusivas como /b/ y /d/ ), como para procesar contrastes fonéticos de poca complejidad en los que se presentan fonemas lentos y di-

\section{Referencias}

Adlard, A., \& Hazan, V. (1998). Speech perception in children with specific reading difficulties (dyslexia). Quarterly Journal of Experimental Psychology: Human Experimental Psychology, 1, 153-177. Recuperado de http://academia.edu/325855/Speech_Perception_In_Children_With_Speci fic_Reading_Difficulties_Dyslexia_

Backes, W., Vuurman, E., Wennekes, R., Spronk, P., Wuisman, M., Van Engelshoven, \& Jolles, J. (2002). Atypical brain activation of reading processes in children with developmental dyslexia. Journal of Child Neurology, 17, 867-71. doi.org/10.1177/08830738020170121601

Best, C.,\& Avery, R. A. (1999). Left hemisphere advantage for click consonants is determined by linguistic significance and experience. Psychological Sciences, 10, 65-70. doi.org/10.1111/1467-9280.00108

Bishop, D.V.M., Carlyon, R.P., Deeks, J.M., \& Bishop, S. J. (1999). Auditory temporal processing impairment: Neither necessary nor sufficient for causing language impairment in children. Journal of Speech Language and Hearing Research, 42, 1295-1310.

Blomert, L.,\& Mitterer, H. (2004). The fragile nature of the speech-perception deficit in dyslexia: Natural vs. synthetic speech. Brain and Language, 8, 21-26. doi.org/10.1016/S0093-934X(03)00305-5

Bradlow, A.R., Kraus, N., Nichol, T.G., McGee, T. J., Cunningham, J., Zecker, S.G.,\& Carrell, T.D. (1999). Effects of lengthened format transition duration on discrimination and neural representation of synthetic CV syllables by normal and learning-disabled children. Journal of the Acoustical Society of America, 106, 2086-2096. doi.org/10.1121/1.427953

Breier, J. I., Gray, L., Fletcher, J. M., Foorman, B. R., \& Klaas, P. (2002). Perception of speech and nonspeech stimuli by children with and without reading disability and attention deficit hyperactivity disorder. Journal of Experimental Child Psychology, 82, 226-250.

Cattell, R. B. y Cattell, A. K. S. (2001). Tests de Factor g. Escala 2. Madrid: T.E.A., Ediciones (Original work published 1950).

Chiappe, P., Chiappe, D. L., \& Siegel, L. (2001). Speech perception, lexicality and reading skill. Journal of Experimental Child Psychology, 80, 58-74. doi.org/10.1006/jecp.2000.2624

Cohen-Mimran, R. (2006). Temporal Processing Deficits in Hebrew Speaking Children with Reading Disabilities. Journal of Speech, Language, and Hearing Research, 49, 127-137. doi. 10.1044/1092-4388(2006/010)

Csépe, V., Gyurkócza, E. E., \& Osman-Sági, J. (1998). Normal and disturbed phoneme perception as reflected by the mismatch negativity: Do eventrelated potentials help to understand dyslexia? Pathophysiology, 5, 202. doi.org/10.1016/S0928-4680(98)81079-1

Cuetos, F., Rodríguez, B., Ruano, E. y Arribas, D. (2007). PROLEC-R. Batería de Evaluación de los procesos lectores - Revisada. Madrid. TEA Ediciones.

de Gelder, B., \& Vroomen, J. (1998). Impaired speech perception in poor readers: Evidence from hearing and speech reading. Brain and Language, 64, 269281. Recuperado de http://www.tilburguniversity.org/Pubs/19.pdf

De Martino, S., Espesser, R., Rey, V., \& Habib, M. (2001). The "Temporal Processing Deficit" hypothesis in dyslexia: New experimental evidence. Brain and Cognition, 46, 104-108. doi.org/10.1016/S0278-2626(01)80044-0

De Weirdt, W. (1988). Speech perception and frequency discrimination for good and poor readers. Applied Psycholinguistics, 9, 163-183. doi.org/10.1017/S0142716400006792

Dufor, O., Serniclaes, W., Sprenger-Charolles, L., \& Démonet, J. F. (2007). Topdown processes during auditory phoneme categorization in dyslexia: A PET $\begin{array}{lll}\text { study. } & \text { NeuroImage, } & \text { 34(4), }\end{array}$ doi.org/10.1016/j.neuroimage.2006.10.034 ferentes en sonoridad, punto y modo de articulación (/f/ y $/ 1 /)$. Por tanto, la diferencia encontrada entre los grupos estaría reflejando dificultades para procesar características temporales de los sonidos del habla.

Agradecimientos.- Agradecemos la colaboración de la Fundación General de la Universidad de la Laguna y de la Unidad de Atención a las Dificultades de Aprendizaje (UADA). Esta investigación ha sido cofinanciada por el Ministerio de Ciencia e Innovación ( ${ }^{\circ}$ ref. PSI2010-15133) y la UADA.

Eden, G.F., \& Zeffiro, T.A. (1998). Neural systems affected in developmental dyslexia revealed by functional neuroimaging. Neuron, 21, 279-82. doi.org/10.1016/S0896-6273(00)80537-1

Ellis S. (2005). Speech Perception in Specific Language Impairment. In David B. Pisoni y Robert E. Remez (eds.), The Handbook of Speech Perception (pp. 567588). Oxford: Blackwell Publishing.

Farmer, M.E., \& Klein, R.M. (1995). The evidence for a temporal processing deficit linked to dyslexia: A review. Psychonomic Bulletin y Review, 2, 460-493. doi.org/10.3758/BF03210983

Faul, F., Erdfelder, E., Lang, A.-G., \& Buchner, A. (2007). G*Power 3: A flexible statistical power analysis program for the social, behavioral, and biomedical $\begin{array}{llll}\text { sciences. Behavior Research Methods, 39, 175-191. } & \end{array}$ doi.org/10.3758/BF03193146

Godfrey, J.J., Syrdal-Lasky, A.K., Millay, K.K., \& Knox, C.M. (1981). Performance of dyslexic children on speech perception test. Journal of Experimental Child Psychology, 32, 401-424. doi.org/10.1016/0022-0965(81)90105-3

Goswami, U., Wang, H.L., Cruz, A., Fosker, T., Mead, N., \& Huss, M. (2011) Language-universal sensory deficits in developmental dyslexia: English, Spanish, and Chinese. Journal of Cognitive Neuroscience, 23, $325-337$. doi.org/10.1162/jocn.2010.21453

Habib, M., Rey, V., Daffaure, V., Camps, R., Espesser, R., Joly Potuzz, B., \& Demonet, J. F. (2002). Phonological training in children with dyslexia using temporally modified speech: A three step pilot investigation. Journal of Language and Communication Disorders, 37, 289-308. doi.org/10.1080/13682820210136278

Hämäläinen, J., Salminen, H., \& Läppanen, P. (2012). Basic auditory processing deficits in dyslexia: Systematic review of the behavioral and event-related potential/field evidence. Journal of Learning Disabilities. Published online February 8, 2012. doi.org/10.1177/0022219411436213.

Hari, R., \& Kiesila, P. (1996). Deficit of temporal auditory processing in dyslexic adults. Neuroscience Letters, 205, 138-140. doi.org/10.1016/03043940(96)12393-4

Jiménez, J. E. (1995). Prueba de conciencia fonémica. En J.E. Jiménez y M.R. Ortiz (Eds.), Conciencia fonológica y aprendizaje de la lectura: Teoría, evaluación e intervención (pp. 74-78). Madrid: Síntesis.

Kinsbourne, M., Rufo, D.T., Gamzu, E., Palmer, R.L., \& Berliner, A. K. (1991). Neuropsychological deficits in adults with dyslexia. Developmental Medicine and Cbild Neurology, 3, 763-775. doi.org/10.1111/j.1469-8749.1991.tb14960.x

Laasonen, M., Virsu, V., Oinonen, S., Sandbacka, M., Salakari, A., \& Service, E. (2012). Phonological and sensory short-term memory are correlates and both affected in developmental dyslexia. Reading and writing, 25, 71 - 108. doi.org/10.1007/s11145-011-9356-1

Lieberman, P. B., Meskill, R. H., Chatillon, M., \& Schupack, H. (1985). Phonetic speech perception deficit in dyslexia. Journal of Speech Language and Hearing Research, 28, 480-486.

Lyon, G.R., Shaywitz, S.E., \& Shaywitz, B.A. (2003). Defining dyslexia, comorbidity, teachers' knowledge of Language and Reading: A definition of dyslexia. Annals of Dyslexia, 53, 1-14.

Maassen, B., Groenen, P., Crul, T., Assman-Hulsmans, C., \& Gabreels, F. (2001). Identification and discrimination of voicing and place-of-articulation in developmental dyslexia. Clinical Linguistics and Phonetics, 4, 319-339.

McAnally, K.I., Hansen, P.C., Cornelissen, P. L., \& Stein, J. F. (1997). Effect of time and frequency manipulation on syllable perception in developmental dyslexics. Journal of Speech Language and Hearing Research, 40, 912-924.

McArthur, G. M., Ellis, D., Atkinson, C. M., \& Coltheart, M. (2008). Auditory processing deficits in children with reading and language impairments: Can 
they (and should they) be treated? Cognition, 107, 946-977. doi.org/10.1016/j.cognition.2007.12.005

McBride-Chang, C. (1996). Models of Speech Perception and Phonological Processing in Reading. Child Development, 67, $1836-1856$. doi.org/10.2307/1131735

Metsala, J. (1997). Spoken word recognition in reading disabled children. Journal of Educational Psychology, 1, 159-169. doi.org/10.1037//0022-0663.89.1.159

Mody, M., Studdert-Kennedy, M., \& Brady, S. (1997). Speech perception deficit in poor readers: Auditory processing or phonological coding? Journal of Experimental Child Psychology, 64, 199-231. doi.org/10.1006/jecp.1996.2343

Ortiz, M.R. y Guzmán, R. (2003). Contribución de la percepción del habla y la conciencia fonémica a la lectura de palabras. Cognitiva, 15, 3-17. doi.org/10.1174/021435503762800914

Ortiz, M.R., Jiménez, J.E., García, E., Guzmán, R., Hernández-Valle, I., Rodrigo, M., ... Hernández, S. (2007). Locus and nature of perceptual phonological deficit in Spanish children with reading disabilites. Journal of Learning Disabilities, 40, 80-92. doi. 10.1177/00222194070400010601

Ortiz, M.R., Jiménez, J.E., Muñetón, M., Rojas, E., Estévez, A., Rodríguez, C., ... Naranjo, F. (2008). Desarrollo de la percepción del habla en niños con dislexia. Psicothema, 20 (4), 678-683. Recuperado de http://www.sld.cu/galerias/pdf/sitios/rehabilitacion-logo/3540.pdf

Pasquini, E. S., Corriveau, K. H., \& Goswami, U. (2007). Auditory processing of amplitude envelope rise time in adults diagnosed with developmental dyslexia. Scientific Studies in Reading, 11, 259-286. doi.org/10.1080/10888430701344280

Paul, I., Bott, C., Heim, S., Wienbruch, C., \& Elbert, T. R. (2006). Phonological but not auditory discrimination is impaired in dyslexia. European Journal of Neuroscience, 24, 2945-2953. doi.org/10.1111/j.1460-9568.2006.05153.x

Ramus, F. (2004). Neurobiology of dyslexia: a reinterpretation of the data. Trends in Neurosciencie, 27, 720-726. doi: 10.1016/j.tins.2004.10.004

Ramus, F., Rosen, S., Dakin, S.C., Day, B. L., Castellote, J. M., White, S., \& Frith, U. (2003). Theories of developmental dyslexia: insights from a multiple case study of dyslexic adults. Brain, 126, 841-865. doi.org/10.1093/brain/awg076

Reed, M. A. (1989). Speech perception and the discrimination of brief auditory cues in dyslexic children. Journal of Experimental Child Psychology, 48, 270-292.

Rey, V., De Martino, S., Espesser, R., \& Habib, M. (2002). Temporal processing and phonological impairment in Dyslexia: Effect of phoneme lengthening on order judgment of two consonants. Brain and Language, 80, 576-591. doi.org/10.1006/brln.2001.2618

Rosen, S. (2003). Auditory processing in dyslexia and specific language impairment: is there a deficit? What is its nature? Does it explain anything? Journal of Phonetics, 31, 50-527. doi.org/10.1016/S0095-4470(03)00046-9

Rosen, S., \& Manganari, E. (2001). Is there a relationship between speech and nonspeech auditory processing in children with dyslexia? Journal of Speech Language and Hearing Research, 44, 720-736. doi.org/10.1044/10924388(2001/057)

Sánchez-Meca, J. (1985). La hipótesis del déficit perceptivo del retraso específico en lectura: Un estudio meta-analítico. Anales de Psicología, 2, 75-91.

Schulte-Koerne, G., Deimel, W., Bartling, J., \& Remschmidt, H. (1999). The role of phonological awareness, speech perception, and auditory temporal processing for dyslexia. European Child and Adolescent Psychiatry, 3, 28-34. doi.org/10.1007/PL00010690

Serniclaes, W., Sprenger-Charolles , L., Carré, R., \& Démonet, J.-F. (2001). Perceptual discrimination of speech sounds in dyslexics. Journal of Speech Language and Hearing Research, 44, 384- 399. doi.10.1044/1092-4388(2001/032)

Share, D. L., Jorm, A. F., Maclean, R., \& Matthews, R. (2002). Temporal processing and reading disability. Reading and Writing, 15, 151-178. Recuperado de http://edu.haifa.ac.il/personal/dshare/Temporal_processing.pdf
Shaywitz, S.E., Morris, R., \& Shaywitz, B.A. (2008). The education of dyslexic children from childhood to young adulthood. Annual Review of Psychology, 59, 451-75.

Steffens, M. L., Eilers, R. E., Gross-Glenn, K., \& Jallad, B. (1992). Speech perception in adult subjects with familial dyslexia. Journal of Speech Language and Hearing Research, 35, 192-200.

Stein, J.F., \& McAnally, K. (1995). Auditory temporal processing in developmental dyslexics. Irish Journal of Psychology, 16, 220-228. doi.org/10.1080/03033910.1995.10558058

Talcott, J. B., \& Witton, C. (2002). A sensory linguistic approach to the development of normal and impaired reading skills. En E.Witruk, A. Friederici, y T. Lachmann (Eds.), Neuropsychology and cognition series. Basic functions of language and language disorders. Dordrecht, The Netherlands: Kluwer Academic Publishers.

Tallal, P. (1980). Auditory temporal perception, phonics, and reading disabilities in children. Brain and Language, 9, 182-198. doi.org/10.1016/0093 934X(80)90139-X

Tallal, P., \& Piercy, M. (1973). Developmental aphasia: Impaired rate of nonverbal processing as a function of sensory modality. Neuropsychologia, 11, 389398. doi.org/10.1016/0028-3932(73)90025-0

Tallal, P., \& Piercy, M. (1974). Developmental aphasia: Rate of auditory processing and selective impairment of consonant perception. Neuropsychologia, 12, 89-93. doi.org/10.1016/0028-3932(74)90030-X

Tallal, P., \& Piercy, M. (1975). Developmental aphasia: The perception of brief vowels and extended stop consonants. Neuropsychologia, 13, 69-74. doi.org/10.1016/0028-3932(75)90049-4

Temple, E. (2002). Brain mechanisms in normal and dyslexic readers. Current Opinion in Neurobiology, 12, 178-83. doi.org/10.1016/S0959-4388(02)00303-3

Tobey, E. A., \& Cullen, J. K. (1984). Temporal integration of tone-glides by children with auditory-memory and reading problems. Journal of Speech Language and Hearing Research, 27, 527-533.

Van Daal, V., \& van der Leij, A. (1992). Computer-based reading and spelling practice for children with learning disabilities. Journal of Learning Disabilities, 25, 186-195. doi.org/10.1177/002221949202500306

Vandermosten, M., Boets, B., Luts, H., Poelmans, H., Wouters, J., \& Ghesquiere, P. (2011). Impairments in speech and nonspeech sound categorization in children with dyslexia are driven by temporal processing difficulties. Research in Developmental Disabilities 32, 593-603.

Vandermosten, M., Boets, B., Luts, H., Poelmans, H., Golestani, N., \& Wouters, J. (2010). Adults with dyslexia are impaired in categorizing speech and nonspeech sounds on the basis of temporal cues. Proceeding of the National Academy of Sciences of the United States of America, 107(23), 10389-10394. doi.org/10.1016/j.ridd.2010.12.015

Werker, J. F., \& Tees, R. C. (1987). Speech perception in severely disabled and average reading children. Canadian Journal of Experimental Psychology, 41, 4861. doi.org/10.1037/h0084150

Wechsler, D. (2005). Escala de Inteligencia Wechsler para niños - IV Edición (WISCIV).Madrid: TEA.

Wolff, P. H., Michel, G. F., \& Ovrut, M. (1990). The timing of syllable repetitions in developmental dyslexia. Journal of Speech and Hearing Research, 33, 281-289.

Wright, B. A., Lombardino, L. J., King, W. M., Puranik, C. S., Leonard, C. M., \& Merzenich, M. M. (1997). Deficits in auditory temporal and spectral resolution in language-impaired children. Nature, 387, 176-178. doi.org/10.1038/387176a0

Zhang, J., \& McBride-Chang, C. (2010). Auditory sensitivity, speech perception, and reading development and impairment. Educational Psychology Review, 22, 323-338. doi.org/10.1007/s10648-010-9137-4

(Articulo recibido: 17-4-2012; revisado: 6-10-2013, aceptado: 19-2-2014) 